\title{
Predictors of pathological complete response to neoadjuvant chemoradiotherapy for esophageal squamous cell carcinoma
}

\author{
Ren-Wen Huang ${ }^{1}$, Yin-Kai Chao ${ }^{2 *}$, Yu-Wen Wen ${ }^{3}$, Hsien-Kun Chang ${ }^{4}$, Chen-Kan Tseng ${ }^{5}$, Sheng-Chieh Chan ${ }^{6}$ \\ and Yun-Hen Liu ${ }^{2}$
}

\begin{abstract}
Backgrounds: In this study, we evaluated the factors associated with a pathologic complete response (pCR) after neoadjuvant chemoradiotherapy (nCRT) for esophageal squamous cell carcinoma (ESCC).

Methods: Pre-nCRT parameters in ESCC patients treated between 1999 and 2006 were analyzed to identify predictors of pCR. All patients received 5-fluorouracil/cisplatin-based chemotherapy and external beam radiation followed by scheduled esophagectomy. Variables were analyzed using univariate and multivariate analyses with PCR as the dependent variable. Estimated $\mathrm{PCR}$ rate was calculated with a regression model.

Results: Fifty-nine (20.9\%) of 282 patients achieved pCR. Univariate analysis identified four patient factors (age, smoking status, drinking history and hypertension), one pre-nCRT parameter (tumor length) as significant predictors of $\mathrm{pCR}$ (all $P<0.05$ ). On multivariate analysis, tumor length $\leq 3 \mathrm{~cm}$ (favorable, odds ratio (OR): $4.85, P=0.001$ ), patient age $>55$ years (favorable, OR: 1.95, $P=0.035$ ), and being a non-smoker (favorable, OR: 3.6, $P=0.003$ ) were independent predictors of $\mathrm{pCR}$. The estimated $\mathrm{pCR}$ rates based on a logistic regression including those three predictors were $71 \%, 35$ to approximately $58 \%, 19$ to approximately $38 \%$, and $12 \%$ for patients with 3, 2, 1 and 0 predictors, respectively.

Conclusion: Age, smoking habit and tumor length were important pCR predictors. These factors may be used to predict outcomes for ESCC patients receiving nCRT, to develop risk-adapted treatment strategies, and to select patients who could participate in trials on new therapies.
\end{abstract}

Keywords: Pathological complete response, Esophageal cancer, Predictors, Neoadjuvant chemoradiotherapy

\section{Background}

Carcinoma of the esophagus is a highly malignant cancer and is generally associated with a poor prognosis [1]. Despite significant advances in surgical and anesthetic techniques, numerous patients develop recurrence after an apparently curative resection [1]. In order to improve resectability and reduce distant metastases, a treatment regime combining chemotherapy, radiotherapy and surgery was introduced in 1995 [2]. A recent meta-analysis has confirmed the benefit of this strategy [3]. However,

\footnotetext{
* Correspondence: chaoyk@cgmh.org.tw

²Division of Thoracic and Cardiovascular Surgery, Chang Gung Memorial Hospital, Linkou, College of Medicine, Chang Gung University, Taoyuan, Taiwan

Full list of author information is available at the end of the article
}

there is growing evidence that neoadjuvant chemoradiotherapy (nCRT) significantly prolongs survival only in patients who have a good response, as measured by a pathological complete response ( $\mathrm{pCR})$, where no residual tumor is found on the resected specimen [4-6]. A pCR occurs in approximately 15 to $30 \%$ of cases, and approximately $60 \%$ of patients in whom pCR is achieved survive beyond five years, irrespective of the actual treatment protocol or tumor histology [4-6]. Conversely, for patients who do not respond to nCRT, the prognosis was even poorer than for patients who received primary surgery [7]. In these cases, valuable treatment time was lost, patients unnecessarily experienced severe CRT toxicity, and may even have lost the opportunity to have potentially curative surgery. Thus, identification of accurate predictors of $\mathrm{pCR}$ 
would be of great value in optimizing outcomes and in the design of clinical trials.

Previous studies have evaluated the use of clinical parameters as potential markers of tumor response to nCRT in esophageal cancer, and in these studies, a lower clinical stage was associated with a higher $\mathrm{pCR}$ rate in the esophageal adenocarcinoma group [8]. Other studies have explored the use of molecular and genetic predictors of $\mathrm{pCR}$. In the study of the latter, microarray technology has been used to investigate the predictive genetic signatures in esophageal cancer, thereby generating classifiers capable of high predictive accuracy [9-12]. However, different studies have generated different sets of markers which do not overlap, and which have not been validated in large-scale human studies [9-12]. Other studies that have attempted to identify specific molecular markers have generally focused on the squamous subtype $[13,14]$. The prognostic impact of these markers was determined indirectly through interactions with other unfavorable pathological descriptors, such as advanced $\mathrm{T}$ stage or lymph node metastases, resulting in their exclusion from multivariate analysis.

Given the lack of success of previous studies in identifying useable predictive markers for $\mathrm{pCR}$ to $\mathrm{nCRT}$ treatment in esophageal squamous cell carcinoma (ESCC), we undertook a thorough analysis based on multiple common clinical parameters in a large number of patients.

\section{Methods}

\section{Patients}

The medical records of 282 esophageal cancer patients who underwent esophagectomy after nCRT were obtained from the institutional review board-approved database from the Chang Gung Memorial Hospital (CGMH) between 1995 and 2012.

Pretreatment staging involved computed tomography (CT) of the chest and abdomen, esophagography and endoscopic ultrasonography (EUS). Pretreatment tumor length was defined as the maximum tumor length measured using a barium contrast agent. The date of the last follow-up was 31 August 2013. Staging was performed according to the American Joint Committee on Cancer (AJCC) 7th edition.

The smoking status of each patient was classified into two categories: never-smokers and ever-smokers (former and current). Similarly, drinking status was also classified into two groups: never-drinkers and ever-drinkers (former and current).

\section{Neoadjuvant CRT and surgery}

The nCRT regimens were as follows. 5-Fluorouracil (5-FU) was administered as a continuous infusion over $96 \mathrm{~h}(1,000$ $\mathrm{mg} / \mathrm{m}^{2}$ per day) from Day 1 to Day 4 and from Day 29 to Day 33. Cisplatin was administered as an intravenous bolus $\left(75 \mathrm{mg} / \mathrm{m}^{2}\right)$ on Day 1 and Day 29. Radiation therapy between Day 4 and Day 29 consisted of a total dose of 30 Gy in 200 cGy daily fractions, administered five days a week. Preoperative radiotherapy encompassed the whole esophagus together with regional lymphatic tissue. The supraclavicular fossa, celiac and pericardial lymphatic regions were also irradiated, unless the dose delivered to normal tissues was poorly tolerated. Radiation was delivered through paired anterior and posterior treatment portals, and the dose was prescribed to the field center.

Restaging was performed between four and six weeks after completion of nCRT. Criteria for surgical eligibility included the following: (i) the patient was physiologically fit for surgery, with no liver cirrhosis (>Child B) or heart failure (New York Heart Association class III, IV); (ii) no evidence of a tracheoesophageal fistula; and (iii) no recurrent laryngeal nerve invasion. The standard surgical approach consisted of a limited thoracotomy on the right side and intrathoracic gastric tube reconstruction (the Ivor-Lewis procedure) for lesions of the middle/ lower-third of the esophagus. Upper-third/cervical lesions were treated with neck anastomosis (the McKeown procedure). Two-field lymph node dissection was performed in all patients, and cervical lymphadenectomy was performed in selected patients who showed evidence of residual disease in the neck. Pyloroplasty and feeding jejunostomy were not routinely performed. A nasogastric tube was placed in each patient until the anastomotic sites were closed, as assessed by esophagography on postoperative Day 14.

Hospital mortality was defined as any death, regardless of cause, occurring (1) within 30 days after surgery in or out of the hospital, and (2) after 30 days during the same hospitalization subsequent to the operation.

\section{Post-therapy surveillance}

After surgery, no adjuvant therapy was given once $\mathrm{pCR}$ had been achieved. All patients underwent chest radiography every three months and CT every six months during the first two years. Panendoscopy was performed if symptoms of recurrent cancer were present. Follow-up data were obtained from medical records and referring physicians, and survival data were updated every six months. The National Cancer Registry Database of Taiwan was used to update missing follow-up information.

\section{Data analysis}

Overall survival (OS) was calculated from the date of surgery to the date of death. Disease-specific survival (DSS) was measured from the date of surgery to the date of known cancer-related death. Pathologic CR was defined as the absence of any tumor cells in the operative pathologic specimen, at the primary site and in lymph node regions. Descriptive statistics were used to describe 
the baseline characteristics, including demographics and pre-nCRT factors of pCR, non-pCR and overall samples. Chi-square tests were performed to determine significant univariate predictors of $\mathrm{pCR}$. All identified significant continuous predictors in univariate analysis were further categorized as corresponding dichotomous variables and each cutoff value was determined by implementing a corresponding binary recursive partition in a conditional inference framework [15]. The criterion in a conditional inference framework is to maximize the 1 minus $P$-value. These were implemented in a non-commercial statistical software R (http://www.R-project.org) with an optional package AUCRF (Random Forest and the Area Under the Curve) and party.

Next, a multivariate logistic regression analysis was then used to identify the significant multivariate predictors of pCR. In a backward, stepwise fashion, the univariate variable $(P<0.1)$ with the least significance was eliminated from the multivariate model. This was continued until only significant variables remained. The final results of a multivariate logistic regression were expressed in odds ratios and confidence intervals. Finally, sstimated pCR rates were obtained by the logistic regression using all possible combinations of significant predictors. Survival curves were plotted using the Kaplan-Meier method. Two-tailed $P$-values $<0.05$ were regarded as significant. All statistical analyses were performed using SPSS 12.0 software (SPSS Inc., Chicago, IL, USA).

\section{Results}

\section{General characteristics of the study participants}

Over the study period, 313 stage II to IV esophageal cancer patients received nCRT followed by surgery in the CGMH. We excluded histology other than squamous cell subtype $(\mathrm{n}=31)$, and 282 patients were finally included in the study. The general characteristics of the entire cohort were summarized in Table 1 . There were 272 males and 10 females with the mean age of 55.2 years (range: 31 to 78 years). Most of the tumors occurred in the middle-third of the esophagus $(58.4 \%, 164 / 282)$. The pretreatment mean tumor length was $6.79 \mathrm{~cm}$ (range: 1.5 to $16 \mathrm{~cm}$ ), as assessed by esophagography. Fifty-nine (20.9\%) of the 282 patients achieved pCR.

The Ivor-Lewis procedure was used in 231 individuals and 48 patients were treated with the Mackeown procedure. Three patients received exploratory surgery without resection due to intraoperatively identified T4 or M1 disease. For reconstruction, the stomach was used in 272 cases and colon interposition in 7 cases. In-hospital mortality occurred in 16 cases.

\section{Survival outcome: $\mathrm{pCR}$ versus non-pCR}

The median OS of the entire population was 18 months (95\% confidence interval (CI): 16.5 to 20 months). At the end of the study period, $50.3 \%$ of pCR patients and $83 \%$ of non-pCR patients had died. The median OS was 98.8 months (95\% CI: 43.7 to 153 months) in pCR patients and 15.5 months (95\% CI: 13.8 to 17.2 months) in non-pCR patients. This difference was statistically significant (Figure 1, $P<0.001$ ).

\section{Univariate analysis of predictive parameters for $\mathrm{pCR}$}

The baseline demographic features differed significantly between groups. As shown in Table 1, the pCR group of patients were on average older, fewer had a history of smoking or alcohol use and more had a history of hypertension (HTN). With respect to the pre-nCRT parameters, patients in the pCR group had a significantly shorter tumor length on average. There were no differences in hemoglobin $(\mathrm{Hb})$ levels, white blood cell (WBC) counts, platelet counts or albumin levels before nCRT. Celiac or lower neck lymph node metastases were more common in the non-pCR group with borderline significance $(P=0.15)$.

\section{Multivariate analysis of predictive parameters for $\mathrm{PCR}$}

In order to improve clinical utility, significant continuous variables selected from univariate analysis (age and tumor length) were further transformed onto an ordinal scale before entering them into a multivariate analysis based on a recursive partition.

The optimal cut-points were 55 for age and 3 for tumor length, respectively (Figure 2A, B).

As shown in Table 2, multivariate analysis identified a tumor length of $\leq 3 \mathrm{~cm}$ (favorable, odds ratio (OR): 2.1, $P=0.02$ ), patient age of $>55$ years (favorable, OR: 2.3 , $P=0.008$ ) and a non-smoking status (favorable, OR: 3.36 , $P=0.003$ ) as independent predictors of pCR while HTN and alcohol use were eliminated. Finally, the estimated pCR rates based on a logistic regression including those three dichotomous predictors were $71 \%, 35$ to approximately $58 \%, 19$ to approximately $38 \%$, and $12 \%$ for patients with 3,2, 1 and 0 predictors, respectively.

\section{Discussion}

Neoadjuvant CRT is currently in widespread use as the first-line treatment for locally advanced esophageal cancer [3]. It has become increasingly evident that only patients with a good response (pCR or near $\mathrm{pCR}$ ) after nCRT have a survival benefit, whereas for those with little response after nCRT, survival is invariably poor [4-7]. In nonresponders, a valuable therapeutic window may have been lost, the patient may have unnecessarily experienced severe CRT toxicity, and may even have lost the opportunity to have potentially curative surgery [7]. Thus, reliable predictive factors for $\mathrm{pCR}$ are of great clinical importance. In the current study, we have analyzed a number of potential predictive factors for $\mathrm{pCR}$ in esophageal cancer of the 
Table 1 Demographic and pre-nCRT clinical factors in pCR and non-pCR groups

\begin{tabular}{|c|c|c|c|c|}
\hline \multicolumn{5}{|c|}{ Demographic factors } \\
\hline Parameters & All $(n=282)$ & $\mathrm{pCR}(\mathrm{n}=59)$ & Non-pCR $(n=223)$ & $P$-value \\
\hline Gender & & & & 0.94 \\
\hline Male & 272 & 57 & 215 & \\
\hline Female & 10 & 2 & 8 & \\
\hline Age & $55.2 \pm 9.8$ & $58.6 \pm 9.5$ & $54.4 \pm 9.7$ & 0.003 \\
\hline BMI & & $22 \pm 3.3$ & $21.5 \pm 3.5$ & 0.3 \\
\hline HTN & & & & $<0.001$ \\
\hline Yes & 28 & 13 & 15 & \\
\hline No & 254 & 46 & 208 & \\
\hline DM & & & & 0.093 \\
\hline Yes & 16 & 6 & 10 & \\
\hline No & 266 & 53 & 213 & \\
\hline Smoking & & & & $<0.001$ \\
\hline Ever & 254 & 46 & 208 & \\
\hline Never & 28 & 13 & 15 & \\
\hline Alcohol & & & & 0.03 \\
\hline Ever & 244 & 46 & 198 & \\
\hline Never & 38 & 13 & 25 & \\
\hline Betal nut chewing & & & & 0.39 \\
\hline Ever & 119 & 22 & 97 & \\
\hline Never & 163 & 37 & 126 & \\
\hline \multicolumn{5}{|c|}{ Pre-nCRT factors } \\
\hline Parameters & All $(n=282)$ & pCR $(n=59)$ & Non-pCR $(n=223)$ & $P$-value \\
\hline Pre-nCRT Hb (g/dl) & $13.2 \pm 1.66$ & $13.1 \pm 1.67$ & $13.2 \pm 1.65$ & 0.55 \\
\hline Pre-nCRT WBC (/ul) & $8,046 \pm 2,967$ & $8,286 \pm 3,698$ & $7,982 \pm 2,748$ & 0.486 \\
\hline Pre-nCRT Albumin (g/dl) & $3.99 \pm 0.48$ & $4 \pm 0.5$ & $3.98 \pm 0.48$ & 0.6 \\
\hline Pre-nCRT Platelet $(1,000 / u l)$ & $278 \pm 98$ & $261 \pm 77$ & $283 \pm 103$ & 0.15 \\
\hline Tumor length $(\mathrm{cm})$ & $6.8 \pm 2.6$ & $5.9 \pm 2.6$ & $7 \pm 2.5$ & 0.003 \\
\hline Tumor grade & & & & 0.08 \\
\hline WD & 14 & 3 & 11 & \\
\hline MD & 199 & 34 & 165 & \\
\hline PD & 69 & 22 & 47 & \\
\hline Clinical stage & & & & 0.29 \\
\hline$\|$ & 71 & 15 & 56 & \\
\hline III & 202 & 44 & 158 & \\
\hline IV & 9 & 0 & 9 & \\
\hline Clinical N status & & & & 0.8 \\
\hline No & 40 & 9 & 31 & \\
\hline Non-No & 242 & 50 & 192 & \\
\hline LN metastases site & & & & 0.15 \\
\hline Non or Mediastinal & 236 & 53 & 183 & \\
\hline Outside mediastinal & 46 & 6 & 40 & \\
\hline
\end{tabular}

BMI, Body mass index; Hb, Hemogobin; HTN, Hypertension; nCRT, Neoadjuvant chemoradiotherapy; pCR, Pathological complete response; WBC, White blood cells. LN,Lymph node; DM, Diabetes mellitus. 


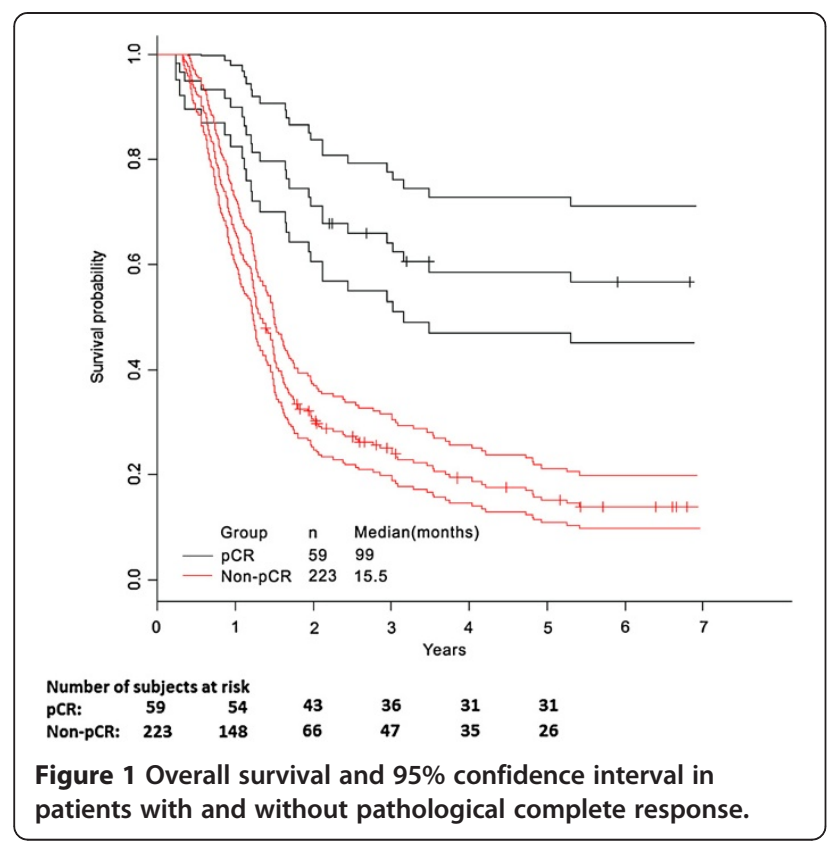

squamous cell carcinoma subtype, treated using a uniform CRT protocol. Although we did not evaluate molecular markers, our study is distinguished by its large size and the incorporation of multiple common clinical parameters. We identified three predictive factors for $\mathrm{pCR}$ - age, smoking status and tumor length - all of which are easily measureable and, hence, easily verifiable in large-scale trials. These factors may ultimately form the basis for a practical predictive marker for routine clinical use.

Smoking is a well-known risk factor for esophageal cancer [16]. However, few reports directly evaluate smoking as a prognostic factor for esophageal cancer, or
Table 2 Multivariate analysis of significant predictors for pathological complete response

\begin{tabular}{lll}
\hline Predictors & OR $\mathbf{( 9 5 \% ~ C l )}$ & $\boldsymbol{P}$-value \\
\hline Ever-smoker & Reference & 0.003 \\
Never-smoker & $3.6(1.55$ to approximately 8.34) & \\
Tumor length $>3 \mathrm{~cm}$ & Reference & 0.001 \\
Tumor length $\leqq 3 \mathrm{~cm}$ & $4.85(1.84$ to approximately 12.76) & \\
Age $\leqq 55$ y/o & Reference & 0.035 \\
Age $>55 \mathrm{y} / \mathrm{O}$ & $1.95(1.05$ to approximately 3.64) & \\
\hline
\end{tabular}

determine whether it can influence the response to CRT $[17,18]$. In our study, we found that smoking was a statistically significant, negative predictor for pCR. Although the mechanism by which smoking reduces the efficacy of CRT is still unknown, overexpression of DNA repair enzymes because of smoking is one possibility. Increased expression of a DNA repair enzyme was reported in heavy smokers with esophageal cancer or non-small cell lung cancers and was also associated with a poor response to CRT $[19,20]$. Taken together, these reports might explain the poor response to CRT in patients with a heavy smoking habit in our study.

The tumor length in esophageal cancer has long been regarded as an important prognostic factor and is consequently one of the T stage descriptors $(<5 \mathrm{~cm}$ : T1; $>5 \mathrm{~cm}$ : T2) in the fifth AJCC edition. However, the importance of tumor length was outweighed by the depth of invasion from 1987 onwards because of their strong inter-correlation [21]. A growing number of studies since then have re-confirmed that tumor length is an indicator of more aggressive behavior independent of $\mathrm{T}$ stage $[22,23]$. Our findings suggest that tumor length might also predict the response to CRT, an observation that could potentially be
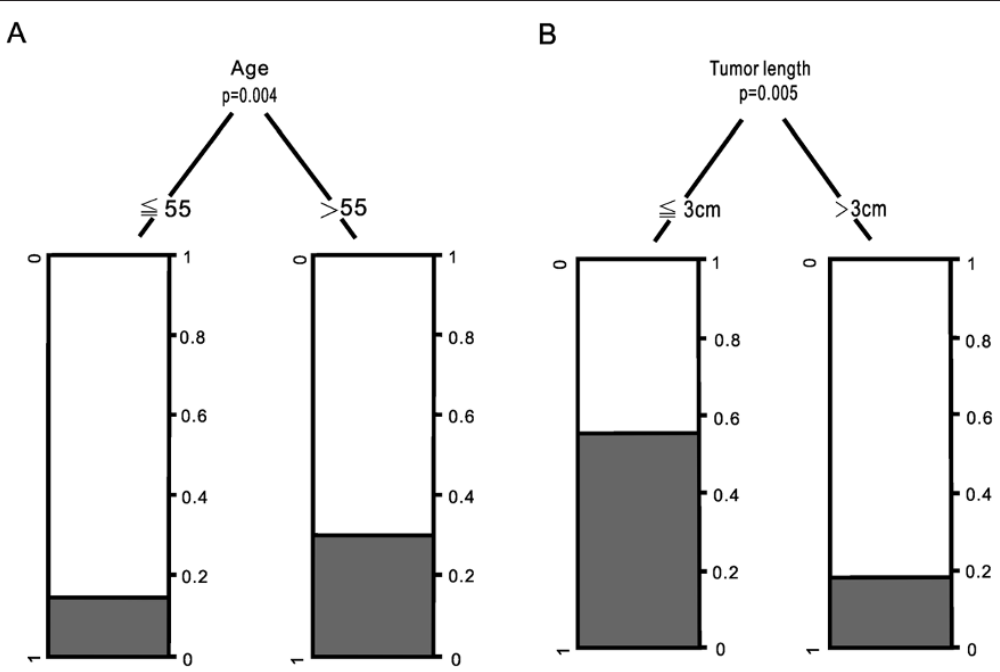

Figure 2 Relative frequencies of $p C R$ and non-pCR groups of the binary classification tree for age (A) and tumor length (B). 
explained by current radiobiological models, the number of cells killed, as described previously in a study on rectal cancer [24].

Old age was not a significant prognostic factor for esophageal carcinoma in most studies but was identified as a predictor for pCR in the current study [25]. Older patients ( $>55$ years) were 1.95 times more likely to achieve pCR than younger patients ( $\leq 55$ years) after nCRT. The reasons for this are unclear and may relate to a complex set of interactions. In Taiwan, earlier ageonset esophageal cancer represents a unique cohort; these patients are more frequently associated with habitual use of substances (tobacco, alcohol or areca nut (seed of the Areca palm)) that are known to reduce the response to radiotherapy [26]. In the current study, we also observed a similar trend of habitual use of substances at a younger age (Additional file 1: Table S1).

Predictive factors can be useful in the design of clinical trials for new therapies and, to a lesser extent, to avoid ineffective therapy. For example, in a cohort where no positive predictor was available, only $12 \%$ could achieve pCR following standard 5FU/cisplatin-based CRT. These patients might be offered a chemotherapy regimen other than $5 \mathrm{FU} /$ cisplatin or received direct surgery whenever curative resection was possible. On the other hand, in patients with multiple predictors, in addition to the routine use of CRT as the first-line treatment, the anticipated high $\mathrm{pCR}$ rate also can lead to the implementation of a trial with organ preservation strategy after nCRT.

We are aware that some weaknesses are inherent in this study. These include the fact that this study was retrospective with a long study period and that it did not incorporate any molecular markers or gene expression profiles. In addition, the radiation dose used in nCRT was lower than that currently used in routine practice (30 Gy vs. 45 to 50.4 Gy), which may have contributed to a lower pCR rate. Future validation of our findings with larger sample sizes and different CRT protocols is therefore required.

\section{Conclusion}

In this study, we showed that older patients ( $>55$ years) who have never smoked and with an initial tumor length of $\leq 3 \mathrm{~cm}$ were more likely to achieve pCR. These factors may be used to predict outcomes for ESCC patients receiving $\mathrm{nCRT}$, to develop risk-adapted treatment strategies, and to select patients who could participate in trials on new therapies.

\section{Additional file}

Additional file 1: Table S1. Demographic and clinical characteristics of patients with different age ( $\leq 55$ or $>55$ years).

\section{Abbreviations}

AJCC: American Joint Committee on Cancer; CGMH: Chang Gung Memorial Hospital; CT: Computed tomography; ESCC: Esophageal squamous cell carcinoma; Hb: Hemoglobin; HTN: Hypertension; nCRT: Neoadjuvant

chemoradiotherapy; OS: Overall survival; pCR: Pathologic complete response; WBC: White blood cells; DM: Diabetes mellitus.

\section{Competing interests}

All authors declare no conflicts of interest.

\section{Authors' contributions}

YKC had full access to all of the data in the study and takes responsibility for the integrity of the data and the accuracy of the data analysis. RWH and YKC contributed to the conception and design of the study, data acquisition, analysis and interpretation of the data, and the writing and revision of the manuscript. YWW contributed to the data analysis and statistical analysis. HKC, CKT, SCC and YHL contributed to quality control of the data and the manuscript review. All authors read and approved the final manuscript.

\section{Acknowledgements}

This study was supported in part by grant NSC101-2314-B-182-094-MY2 from the National Science Council, Executive Yuan, and in part by grant NMRPD1B1431 from the Chang Gung University, Taiwan, Republic of China.

\section{Author details}

'Department of Plastic and Reconstructive Surgery, Chang Gung Memorial Hospital, Linkou, College of Medicine, Chang Gung University, Taoyuan, Taiwan. ${ }^{2}$ Division of Thoracic and Cardiovascular Surgery, Chang Gung Memorial Hospital, Linkou, College of Medicine, Chang Gung University, Taoyuan, Taiwan. ${ }^{3}$ Clinical Informatics and Medical Statistics Research Center, Chang Gung University, Taoyuan, Taiwan. ${ }^{4}$ Division of Hematology/Oncology, Chang Gung Memorial Hospital, Linkou, College of Medicine, Chang Gung University, Taoyuan, Taiwan. ${ }^{5}$ Department of Radiation Oncology, Chang Gung Memorial Hospital, Linkou, College of Medicine, Chang Gung University, Taoyuan, Taiwan. ${ }^{6}$ Department of Nuclear Medicine, Chang Gung Memorial Hospital, Keelung, College of Medicine, Chang Gung University, Taoyuan, Taiwan.

Received: 27 January 2014 Accepted: 6 May 2014

Published: 29 May 2014

\section{References}

1. Enzinger PC, Mayer RJ: Esophageal cancer. N Engl J Med 2003, 349:2241-2252.

2. Walsh TN, Noonan N, Hollywood D, Kelly A, Keeling N, Hennessy TP: A comparison of multimodal therapy and surgery for esophageal adenocarcinoma. N Engl J Med 1996, 335:462-467.

3. Sjoquist KM, Burmeister BH, Smithers BM, Zalcberg JR, Simes RJ, Barbour A, Gebski V: Survival after neoadjuvant chemotherapy or chemoradiotherapy for resectable oesophageal carcinoma: an updated meta-analysis. Lancet Oncol 2011, 12:681-692.

4. Berger AC, Farma J, Scott WJ, Freedman G, Weiner L, Cheng JD, Wang H, Goldberg M: Complete response to neoadjuvant chemoradiotherapy in esophageal carcinoma is associated with significantly improved survival. J Clin Oncol 2005, 23:4330-4337.

5. Donahue JM, Nichols FC, Li Z, Schomas DA, Allen MS, Cassivi SD, Jatoi A Miller RC, Wigle DA, Shen KR, Deschamps C: Complete pathologic response after neoadjuvant chemoradiotherapy for esophageal cancer is associated with enhanced survival. Ann Thorac Surg 2009, 87:392-398. discussion 398-399.

6. Meredith KL, Weber JM, Turaga KK, Siegel EM, McLoughlin J, Hoffe S, Marcovalerio M, Shah N, Kelley S, Karl R: Pathologic response after neoadjuvant therapy is the major determinant of survival in patients with esophageal cancer. Ann Surg Oncol 2010, 17:1159-1167.

7. Dittrick GW, Weber JM, Shridhar R, Hoffe S, Melis M, Almhanna K, Barthel J, McLoughlin J, Karl RC, Meredith KL: Pathologic nonresponders after neoadjuvant chemoradiation for esophageal cancer demonstrate no survival benefit compared with patients treated with primary esophagectomy. Ann Surg Oncol 2012, 19:1678-1684.

8. Rohatgi P, Swisher SG, Correa AM, WU TT, Liao Z, Komaki R, Walsh GL, Vaporciyan AA, Rice DC, Roth JA, Ajani JA: Characterization of pathologic complete response after preoperative chemoradiotherapy in carcinoma 
of the esophagus and outcome after pathologic complete response. Cancer 2005, 104:2365-2372.

9. Schauer M, Janssen KP, Rimkus C, Raggi M, Feith M, Friess $H$, Theisen J: Microarray-based response prediction in esophageal adenocarcinoma. Clin Cancer Res 2010, 16:330-337.

10. Maher SG, Gillham CM, Duggan SP, Smyth PC, Miller N, Muldoon C, O'Byrne KJ, Sheils OM, Hollywood D, Reynolds JV: Gene expression analysis of diagnostic biopsies predicts pathological response to neoadjuvant chemoradiotherapy of esophageal cancer. Ann Surg 2009, 250:729-737.

11. Duong C, Greenawalt DM, Kowalczyk A, Ciavarella ML, Raskutti G, Murray WK, Phillips WA, Thomas RJ: Pretreatment gene expression profiles can be used to predict response to neoadjuvant chemoradiotherapy in esophageal cancer. Ann Surg Oncol 2007, 14:3602-3609.

12. Luthra R, Wu T, Luthra MG, Izzo J, Lopez-Alvarez E, Zhang L, Bailey J, Lee JH, Bresalier R, Rashid A, Swisher SG, Ajani JA: Gene expression profiling of localized esophageal carcinomas: association with pathologic response to preoperative chemoradiation. J Clin Oncol 2006, 24:259-267.

13. Shimada H, Hoshino T, Okazumi S, Matsubara H, Funami Y, Nabeya Y, Hayashi H, Takeda A, Shiratori T, Uno T, Ito H, Ochiai T: Expression of angiogenic factors predicts response to chemoradiotherapy and prognosis of oesophageal squamous cell carcinoma. Br J Cancer 2002, 86:552-557.

14. Warnecke-Eberz U, Metzger R, Miyazono F, Baldus SE, Neiss S, Brabender J, Schaefer H, Doerfler W, Bollschweiler E, Dienes HP, Mueller RP, Danenberg $\mathrm{PV}$, Hoelscher AH, Schneider PM: High specificity of quantitative excision repair cross-complementing 1 messenger RNA expression for prediction of minor histopathological response to neoadjuvant radiochemotherapy in esophageal cancer. Clin Cancer Res 2004, 10:3794-3799.

15. Hothorn T, Hornik K, Zeileis A: Unbiased recursive partitioning: a conditional inference framework. J Comput Graph Stat 2006, 15:651-674.

16. Oze I, Matsuo K, Ito H, Wakai K, Nagata C, Mizoue T, Tanaka K, Tsuji I, Tamakoshi A, Sasazuki S, Inoue M, Tsugane S, Research Group for the Development and Evaluation of Cancer Prevention Strategies in Japan: Cigarette smoking and esophageal cancer risk: an evaluation based on a systematic review of epidemiologic evidence among the Japanese population. Jpn J Clin Oncol 2012, 42:63-73.

17. Shitara K, Matsuo K, Hatooka S, Ura T, Takahari D, Yokota T, Abe T, Kawai H, Tajika M, Kodaira T, Shinoda M, Tajima K, Muro K, Tanaka H: Heavy smoking history interacts with chemoradiotherapy for esophageal cancer prognosis: a retrospective study. Cancer Sci 2010, 101:1001-1006.

18. Sundelof M, Lagergren J, Ye W: Patient demographics and lifestyle factors influencing long-term survival of oesophageal cancer and gastric cardia cancer in a nationwide study in Sweden. Eur J Cancer 2008, 44:1566-1571.

19. Nozoe T, Korenaga D, Kabashima A, Sugimachi K: Smoking-related increase of O(6)-methylguanine-DNA methyltransferase expression in squamous cell carcinoma of the esophagus. Cancer Lett 2002, 184:49-55.

20. Ohba T, Kometani T, Shoji F, Yano T, Yoshino I, Taguchi K, Kuraoka I, Oda S, Maehara Y: Expression of an X-family DNA polymerase, pol lambda, in the respiratory epithelium of non-small cell lung cancer patients with habitual smoking. Mutat Res 2009, 677:66-71.

21. Sobin $L H$, Hermanek $P$, Hutter RV: TNM classification of malignant tumors. A comparison between the new (1987) and the old editions. Cancer 1988, 61:2310-2314.

22. Wang BY, Goan YG, Hsu PK, Hsu WH, Wu YC: Tumor length as a prognostic factor in esophageal squamous cell carcinoma. Ann Thorac Surg 2011, 91:887-893.

23. Eloubeidi MA, Desmond R, Arguedas MR, Reed CE, Wilcox CM: Prognostic factors for the survival of patients with esophageal carcinoma in the U.S.: the importance of tumor length and lymph node status. Cancer 2002, 95:1434-1443.

24. Willett CG, Warland G, Coen J, Shellito PC, Compton CC: Rectal cancer: the influence of tumor proliferation on response to preoperative irradiation. Int J Radiat Oncol Biol Phys 1995, 32:57-61.
25. Turkyilmaz A, Eroglu A, Subasi M, Karaoglanoglu N: Clinicopathological features and prognosis of esophageal cancer in young patients. Is there a difference in outcome? Dis Esophagus 2009, 22:211-215.

26. Lin MY, Chen MC, Wu IC, Wu DC, Cheng YJ, Wu CC, Chai CY, Lee JM, Wu MT: Areca users in combination with tobacco and alcohol use are associated with younger age of diagnosed esophageal cancer in Taiwanese men. PLoS One 2011, 6:e25347.

doi:10.1186/1477-7819-12-170

Cite this article as: Huang et al.: Predictors of pathological complete response to neoadjuvant chemoradiotherapy for esophageal squamous cell carcinoma. World Journal of Surgical Oncology 2014 12:170.

\section{Submit your next manuscript to BioMed Central and take full advantage of:}

- Convenient online submission

- Thorough peer review

- No space constraints or color figure charges

- Immediate publication on acceptance

- Inclusion in PubMed, CAS, Scopus and Google Scholar

- Research which is freely available for redistribution

Submit your manuscript at www.biomedcentral.com/submit
C Biomed Central 